\title{
Environmental product information (EPI) and LCA
}

\author{
Ittingen-berne, Switzerland, June 22, 2010
}

Geneviève Doublet • Niels Jungbluth

Received: 22 July 2010 /Accepted: 21 November 2010 /Published online: 2 December 2010

(C) Springer-Verlag 2010

\begin{abstract}
The 41st discussion forum addressed different concepts of environmental product information (EPI). The goal was on the one hand to discuss EPI in a theoretical perspective, addressing issues on functional unit and use phase, LCIA methods, and comprehensiveness of environmental indicators. On the other hand, practical examples were presented to show the heterogeneity and challenges in the actual implementation of EPI in Europe and evaluate how far such case studies can be generalized in order to establish one type on environmental information for all types of products. The discussion started with three talks dedicated to Swiss perspectives on EPI, presenting expectations of the Swiss Federal Office for the Environment followed by the results of a Swiss feasibility study investigating a possible concept for EPI, and ending with discussing EPI from a psychological perspective. After three presentations considering different approaches developed in neighboring countries (France, Austria, and Italy) for providing life cycle assessment-based environmental information for products, six short presentations were held covering industry application and case studies. The following issues were addressed during the discussion: the real demand for EPI from the business, the integration of the use phase in the information provided, the questions and comparisons to be addressed with an EPI, the indicators to use in EPI, the effects of EPI on consumers, and the attitude of consumers regarding quantitative environmental indicators.
\end{abstract}

G. Doublet $\cdot$ N. Jungbluth $(\square)$

ESU-services Ltd,

Kanzleistrasse 4,

8610, Uster, Switzerland

e-mail: jungbluth@esu-services.ch
Keywords Comparability · Consistency · Cradle-to-basket · Credibility · Ecological consumer decisions - Ecological time unit. Environmental product declaration .

Environmental product information . Functional unit . Levels of decision making - Transparency - Use phase

\section{Introduction}

In the recent past, several initiatives were launched to show the carbon footprint or other life cycle-based environmental information for consumer products (EPI). While the provision of EPI may contribute to more informed purchase decisions and would help to increase the attention of companies towards environmentally more efficient products and production processes, several key aspects still need clarification.

Firstly, the definition and expectations of EPI are broad, ranging from environmental product declaration for building materials or a city's electricity mix products to desired EPI on food products. Secondly, the level of decision making addressed by EPI differs according to the choices that are addressed. Thirdly, life cycle assessment (LCA)based EPI is confronted with high uncertainties and variable results depending on the definition of the use and end-of-life phase and the LCA methods chosen. Thus, results from EPI might not be comparable between different approaches.

Hence, the 41st discussion forum presented an overview on EPI from theoretical and practical points of view. Expectations from the Swiss Federal Office for the Environment (FOEN) were shown followed by a scientific feasibility study and a psychological study on EPI. Participants became familiar with the use and the conception of EPI in other countries like France, Austria, and Italy. 
Finally, industry implementation and case studies were presented. The goal of the 41st DF was to discuss EPI in Switzerland and neighboring countries in order to increase participants' awareness of the strengths and weakness, the opportunities and the limits of environmental product information in detail.

\section{Swiss perspectives on EPI}

The first session was dedicated to Swiss perspectives on EPI containing three presentations on expectations regarding EPI, feasibility and challenges for providing EPI, and EPI from a psychological point of view.

Anna Wälty (FOEN) outlined the expectations of the FOEN regarding environmental product information. At the beginning, she focused on today's situation showing that consumption decisions need meaningful EPI. A lot of information are provided on quantity, quality, and price, but there are few ecological product information apart from labels and statement of origin. However, there is a demand for better product information on national and international levels that is only achievable by increasing market's transparency. The second part of the presentation focused on what is needed to enhance the market's transparency. Data gathering, data quality, methodologies, and rules to present the environmental impacts must be defined to ensure relevant and comparable data. In the third part of the presentation, the requirements of the EPI for a "True and Fair view" were presented. The first requirement considers the relevance of the EPI with the decisions to be influenced, and the second one sets that information should reflect the reality as close as possible by including all relevant environmental impacts based on a life-cycle approach. These two requirements need prerequisites, namely reliability (third-party review), transparency (traceability), comprehensibility, and consistency. The presenter outlined that collaborations between science, government, and business are mandatory to enhance the market's transparency and ecological consumption behaviors.

Niels Jungbluth (ESU-services Ltd.) presented the feasibility and challenges for providing environmental product information based on LCA. EPI should quantify environmental impacts of products over the life cycle and inform consumers about it in order to support sustainable consumption. The presenter highlighted the special issue concerning the consideration of the use and end-of-life phase in the life cycle inventory assessment. These phases may be very important, depending on the type of product. However, the use phase often shows a high variability, and different end-of-life options exist. Furthermore, the use and the waste management of a product can only partly be influenced by the producer. Therefore, he recommended showing environmental impacts in the first instance from cradle-to-basket, which is also in line with other information like the price of the product. One has to decide also about the level of decision making addressed by the approach and thus about the functional unit for which information can be shown. The presentation evaluated which life cycle impact assessment method is the most appropriate. The Swiss ecological scarcity 2006 method was recommended for EPI in Switzerland. Communication issues were presented, and the need for a communication concept was emphasized. The presenter introduced the Swiss ecological time unit as a possible solution for communicating environmental impacts. He emphasized that clear procedure and guidance are necessary.

Christina Tobler (ETH Zürich) focused on environmental product information from a psychological point of view. A study on consumer's environmental assessment was presented. It showed that there exists high variability between the public's perception and the expert rating of products. Moreover, the results illustrated that many ecolabels lack transparency for the consumer. The evaluability principle was introduced. Preference reversals occur between joint and separate evaluations when a particular attribute is easily evaluated while another is relatively hard to evaluate. Even very important attributes may not be used unless they can be precisely translated into a frame of reference. In the last part, the difference between promotion and prevention was outlined. The results illustrated that negative labels could influence consumers with intermediate environmental concern. It was concluded that consumers need more information on ecological consumption, preferably with a reference frame. The presenter emphasized that legislation is needed to implement a simple and meaningful communication tool to ensure credibility and foster ecological consumption.

\section{Concepts developed in other countries}

In the second part of the discussion forum, it was shown how environmental product information concepts are developed in France, Austria, and Italy.

The planned presentation by Jenny Teufel (Oko-Institut, Germany) on product carbon footprinting and whether or not it is a suitable way for fostering the consumption of products with low $\mathrm{CO}_{2}$ emissions was canceled.

Gérard Gaillard (Agroscope Reckenholz-Tänikon Research Station ART) focused on the environmental product information in France and more precisely on the legal context and the technical challenges. In the first part of the presentation, he showed that the environmental product declaration (EPD) in France is still at the planning phase and an experimental phase should start in July 1, 2011. It has been organized as a participative process involving 12 -sector working groups, a 
methodological working group, and a labeling format working group, which are brought together with the database governance committee in the general platform, which makes decisions and acts as a coordinating body. Life cycle analysis approach focusing on carbon equivalents will be used in parallel with other pertinent indicators depending on the products' category. The French Environment and Energy Agency steers the development and the maintenance of the software, leads and finances the production and management of data, and assists the use of data tools. The example of the database agri-BALYSE for agricultural products was presented. A classification has been made between plant production, animal production, and tropical crops, and simulation models will be used for estimating direct emissions. At the end, the presenter drew attention on the challenge raised by the participative approach involving 1,000 experts with specific interests.

Eva Burger (Sustainable Europe Research Institute (SERI), Austria) talked about Austrian initiatives on EPI. Robust data and indicators are requirements for target setting and monitoring of sustainability strategy. The project developed by theSERI has defined an indicator set including carbon footprint, water rucksack, actual land use, abiotic material input, and biotic material input. This indicator set has been chosen because it is applicable for the whole life cycle of all products and services, it gets down to the root of the environmental issues, considers the main environmental categories, and the scarcity of all natural resources. Four initiatives are shown in implementing EPI. The first one is called "Sustainable Week" and focuses on advocating sustainable buying decisions by explicitly attracting interest in ecological and social sustainable products by means of a common label. The second initiative's objective is to provide carbon footprint information to consumers while the third one named "Back to roots" is similar to the second one, but the carbon footprint is communicated as the relative difference between a conventional product and the "bio-premium" product. The last two initiatives require a standard method for carbon footprint calculations. The fourth initiative is the development of a sustainability label for REWE, one of the leaders in German and European trading and travel. The label estimates biotic, abiotic, water, carbon footprint, and area footprint per kilogram of product. The current layout of the project does not include weighting factors to end with a single score calculation. At the end, the presenter raised some challenges regarding the sustainability at REWE such as the trade-off between high environmental objectives and practicability, the lack of third-party review, and the doubts about the consumer's understanding of the concept.

In the last presentation of the morning session, Leo Breedveld (2B, Italy) showed the developments of product category rules (PCR) and EPD and experiences made in Italy on furniture and other products. The presenter started with the statement that $76 \%$ of the consumers think that companies don't tell the truth in their advertisements. There is a need for a transparent, comparable, and scientifically sound communication. EPD is beneficial by increasing credibility through third-party review, consistency through LCA studies, comparability for improvement, and transparency. The presenter emphasized that EPD is not a marketing or compulsory tool or a guarantee of high environmental performance. It aims to communicate the environmental performance of a company's product. Then, EPD systems developed by Sweden, Norway, Japan, and South Korea were introduced. The example of the assessment of Italian chairs within the Norwegian EPD system called NHO system was shown. The next example was the assessment of mineral water with the Swedish EPD system International EPD Consortium (IEC). These two examples showed the differences between NHO and IEC. Several hundreds of PCR have been developed already, but often they are only applied for one product. It was concluded that EPD should be considered as the beginning of a journey of continuous improvement even though it is still in its childhood. Italy is well presented in international EPD schemes with a main application in building materials. It was finally outlined that comparison between EPD remains difficult due to variations in system boundaries and used data sources.

During the break, participants could get familiar with the Swiss ecological time unit introduced during Niels Jungbluth's presentation. Geneviève Doublet introduced this idea with a poster. The indicator compares environmental impacts with the Swiss political target for 1 year and takes this time as a reference unit. Beverages and snacks had been assessed and results were summarized on a menu. On the buffet table, etiquettes were placed in front of each beverage and snack to catch participants' attention. During the lunch, the desert with a high eco-time puzzled some participants.

\section{Short presentations of experiences with LCA-based environmental information on products}

The first afternoon session was dedicated to six short presentations. The first from Sebastien Humbert (Quantis) claimed the importance to consider a functional unit and the use phase instead of the product in the basket. When comparing instant coffee with drip filter coffee, the choice between the amount of coffee per kilogram and per cup reversed the results while the environmental impacts per cup of filter coffee and capsule espresso coffee are reversed when the use phase is included. He concluded that the use 
phase must be included and the functional unit must be appropriately defined to avoid any distorted results.

Guy Castelan (PlasticsEurope, France) presented the eco-footprint concept. The results of a workshop involving PlasticsEurope, three value chains players, six academia, an EU institution, and member companies showed the need for transparent weighting factors and the possibility to adjust them according to their preferences and objectives. A prototype is being developed. It was concluded that ecofootprint is to be implemented on market by value chain players as a European multi-stakeholder support for decision.

The next presentation by Sébastien Lasvaux (Centre Scientifique et Technique du Bâtiment, France) showed the current practices and perspectives regarding the use of EPD and generic life cycle inventory (LCI) databases to assess the environmental impacts of buildings in France. Up to date, the use of ecoinvent datasets and the French EPD database INIES leads to heterogeneity in results and idle comparison. The outlines of a Ph.D. thesis were given for developing a simplified LCA (sLCA) database. Three outputs from this sLCA were given. First, assess the relevance of simplification in LCI of construction products and materials, second, inter-compare generic and EPD data for a given material, and third, do LCA case studies of buildings with simplified but transparent LCI results and life cycle impact indicators. It was concluded that the development of the growing INIES database of EPD in the building industry would not only support pathways of simplification but also provide additional knowledge.

Zhang Qi (Nestlé) then illustrated the three key objectives of Nestlé for EPD. Firstly, the establishment of scientifically reliable and uniform environmental assessment methodologies for food and drinks, secondly, the identification of suitable tools and guidance for voluntary environmental communication to consumers and other stakeholders, and thirdly, the promotion of continuous environmental improvement measures along the entire food supply chain.

Frank Werner (Werner Environment \& Development) presented experiences of the Institut Bauen \& Umwelt (IBU) scheme for construction products to highlight key factors for a successful EPD. He showed the IBU EPD scheme, which focuses on a business-to-business communication by describing the life cycle of the construction product and the environmental impact assessments and some testing results. Reliable and independently verified information supported by governmental agencies and manufacturers, which are published in one document gathering environmental together with technical and health-related information, are key factors for success. It was concluded that EPD data are easily integrated into tools for building assessments and certification schemes.
Roland Hischier (ecoinvent Center) introduced the requirements of EPI systems and their fulfillment by the ecoinvent database. Common requirements of EPI include life cycle approach over the supply chain, reliable, verified, and comparable data across and outside Europe, and common allocation rules. The ecoinvent database is adjusting itself to satisfy carbon footprint allocation rules, include new sectors and new areas, and add some parameterization tools on geography, time, technologies, and markets. He concluded that higher quality standards would ensure more transparent, well-documented, and well-validated data.

\section{Industry implementation and case studies}

The second part of the afternoon was opened by Gerhard Emch (EWZ Zürich) showing environmental product information for consumers about energy products sold in the City of Zürich. Customers are asking for environmental indicators; therefore, EWZ has decided to publish EPD. He introduced EWZ's intentions to give the same information for the same product, use the same methodology, and cover all aspects of ecologically produced energy. The Swiss energy certificate system certifies renewable electricity products, such as hydro, biomass, wind and solar, either as "naturemade star" or "naturemade basic". He presented the creation of the EPD starting with the definition of the electricity mix followed by the estimation of the grid losses and the emissions of sulfur hexafluoride, then the quantification of waste from waste incineration, and finally, the list of events in Swiss nuclear plants. Inventory data from the ecoinvent database are then assessed with the Swiss ecological scarcity method. The values are given per kilowatt hour and are differentiated between energy qualities sold and procured (produced and bought). The presenter outlined that the EPD is only valid for 1 year due to possible change in the electricity mix and highlighted that a personal contact is required to provide additional comments since the interpretation of both certified electricity mix is difficult. The presenter concluded that EWZ is continuously developing new energy sources for new applications.

In the last presentation of the day, Lionel Cretegny (FOEN) showed criteria for energy-efficient and lowemission vehicles (KEEF, Umweltetikette). He introduced the energy label developed in 2002 consisting only of the car's weight, the fuel consumption, and the $\mathrm{CO}_{2}$ emissions. However, the energy label had serious drawbacks including the fuel consumption per weight. In 2007, new criteria were set concerning climate change, noise pollution, air pollution, and fuel consumption. Today's legislation ordinance project on environmental label was then presented. It is based on a new passenger car with a maximum of nine 
seats, around 3,500 kg, not matriculated, and with less than $2,000 \mathrm{~km}$. The label contains the energy consumption, the $\mathrm{CO}_{2}$ emissions from tank to wheel, the ecopoints score per kilometer based on the ecological scarcity method 2006 and defined ecofactors for all air pollutants. The new passenger cars are classified into seven categories with an increasing share of ecopoints. The presenter concluded that the draft ordinance had been lately refused.

\section{Discussion}

The general discussion was guided by Kathrin Schlup (FOEN) with all the main presenters. The most controversial discussion point was on which system boundaries should be used. A part of the participants argued against the integration of the use phase, which contains too much uncertainty, would lead to double counting of impacts, gives a high workload for developing PCR, and is not suitable for evaluating the achievements in the production chain. Other participants in the audience considered it important to include the full life cycle in EPI because they consider it to be more suited for detailed product comparisons and for guidance of consumer decisions. Also, the level of decision making addressed and thus the questions to be answered by EPI need more clarification. Many perceive EPI as a means for detailed comparisons of products while others would like to see it more as an instrument to guide consumers for these decisions, which are really relevant from an overall point of view. Then, the public's attitude toward EPI was discussed. Some were confident about communicating quantitative indicators while others mentioned that consumers neglect actual labeling of energy content of products and that EPI might assist only consumers with already a strong environmental concern. Many consider carbon footprint alone as not sufficient for an environmental assessment, but it was difficult to conclude as to what would be the most appropriate environmental indicator and how to agree on international standards for this.

All presentations of the discussion forum and the poster about the ecological time assessment of the food offered are available for download at http://www. lcaforum.ch/Downloads/DF41/tabid/79/Default.aspx. 